\title{
MS35-P35 | EFFECT OF CRYSTALlizATION CONDITIONS ON DIURETIC CLOPAMIDE AND ITS COPPER(II) COMPLEXES
}

Gál, Gyula Tamás (Hungarian Academy of Sciences Research Centre for Natural Sciences, Budapest, HUN); May, Nóra V. (Hungarian Academy of Sciences Research Centre for Natural Sciences, Budapest, HUN); Trif, László (Hungarian Academy of Sciences Research Centre for Natural Sciences, Budapest, HUN); Bombicz, Petra (Hungarian Academy of Sciences Research Centre for Natural Sciences, Budapest, HUN)

Clopamide (4-chloro-N-2,6-dimethylpiperidin-1-yl)-3-sulfamoylbenzamide) is an active pharmaceutical ingredient (API) categorized as a thiazide-like diuretic and is used against hypertension and oedema. The carbonyl oxygen and the piperidine nitrogen of the clopamide molecule are able to coordinate to metal centres. The sort of coordination of the bioligand to metal ions can affect their biological properties. Despite of its medical application the structure of the clopamide molecule or any of its metal complexes has not been published so far.

Our primarily aim was to reveal the single crystal structure of clopamide. The outcome of several successful crystallization experiments allowed us to harvest single crystals of a series of clopamide containing compounds. It has opened up the way to determine how far the molecular conformation and the packing arrangement isostructurality - can be preserved in respond to chemical changes to enrich the knowledge on the aspects which contribute to the development of materials with specific properties.

We have studied the free ligand and its copper(II) complexes under different conditions ( $\mathrm{pH}$, solvent, counter ion) applying different techniques (single crystal X-ray diffraction (SXRD), electron paramagnetic resonance (EPR) spectroscopy, and thermoanalytical methods).

SXRD was used to determine the crystal structure of the free ligand and of the bis-ligand copper complexes obtained under different crystallization conditions. We succeeded to determine the crystal structure of both clopamide hemihydrate $(\mathrm{C} 2 / \mathrm{c})$ and ahnydrous clopamide $\left(P 2_{1} / \mathrm{n}\right)$. The bis-ligand copper(II) complexes of clopamide have been crystallized from different $\mathrm{pH}$ 's and solvents.

Acta Cryst. (2019). A75, e590 